\title{
Investigating the effectiveness of advertising on declining social networks
}

\section{GERGELY KOCSIS and IMRE VARGA}

\begin{abstract}
.
In this paper we aim to investigate the question if it's worth it to advertise on declining social networks or not. Our investigations are based on computer simulations using a previously defined and here simplified model of information spreading. To make our results as close to the real life as possible we run simulations both on a real network sample and on several generated networks representing as well classical scale-free-like social topologies as declining social networks. As a result we found how the continuous destruction of the network affects the spreading, changing also the effectiveness of the advertising.
\end{abstract}

Acknowledgement. The publication was supported by the TÁMOP-4.2.2.C-11/1/KONV-2012-0001 project. The project has been supported by the European Union, co-financed by the European Social Fund.

\section{REFERENCES}

[1] Albert, R. and Barabási, A.-L., Statistical mechanics of complex networks, Rev. Mod. Phys., 74, 47-97 (2002)

[2] Albert, R. and Barabási, A.-L., Emergence of Scaling in Random Networks, Science 286 5439, 509-512 (1999)

[3] Albert, R., Jeong, H. and Barabási, A. L., The Internet's Achilles' Heel: Error and attack tolerance of complex networks, Nature 406, 378-382 (2000)

[4] Caldarelli, G., Capocci, A., De Los Rios, P. and Muoz, M. A., Scale-Free Networks from Varying Vertex Intrinsic Fitness, Phys. Rev. Lett., 89, 258702 (2002)

[5] Dorogovtsev, S. N. and Mendes, J. F. F., Effect of the accelerating growth of communications networks on their structure, Phys. Rev. E, 63, 025101 (2001)

[6] Gjoka, M., Kurant, M., Butts, C. T. and Markopoulou, A., Walking in Facebook: A Case Study of Unbiased Sampling of OSNs, Proceedings of IEEE INFOCOM '10, San Diego, CA, (2010)

[7] Kocsis, G. and Kun, F., Competition of information channels in the spreading of innovations, Phys. Rev. E, 84, 026111 (2011)

[8] Kosmidis, K. and Bunde, A., Propagation of confidential information on SF networks, Phys. A, 376, 699-707 (2007)

[9] Lee, H. Y., Chan, H. Y. and Hui, P. M., Scale-free networks with tunable degree distribution exponents, Phys. Rev. E, 69, 067102 (2004)

[10] Tang, X. G. and Wong, E. W. M., Information traffic in scale-free networks with fluctuations in packet generation rate, Phys. A, 388, 4797-4802 (2009)

[11] Varga, I., Németh, A. and Kocsis, G., A novel method of generating tunable underlaying network topologies for social simulation, submitted to The 4th IEEE International Conference on Cognitive Infocommunicaitons (2013)

[12] Varghese, B. M., The Life Cycle of a Social Network, Techpedia (http:/ / www.techipedia.com/2011/social-network-life-cycle/ - last visited: June 20, 2013) (2011)

DEPARTMENT OF INFORMATICS SYSTEMS AND NETWORKS

UNIVERSITY OF DEBRECEN

26 Kassai Út, Debrecen, Hungary

E-mail address: kocsis.gergely@inf.unideb.hu

E-mail address: varga.imreeinf.unideb.hu

Received: 04.11.2013; In revised form: 05.06.2014; Accepted: 12.06.2014

2010 Mathematics Subject Classification. 05C82, 90B15, 91D30.

Key words and phrases. Scale-free networks, social networks information spreading, simulation, modeling. 